\title{
Reconstrucción de la cobertura de la vegetación y uso del suelo hacia 1550 y sus cambios a 2007 en la ecorregión de los bosques valdivianos lluviosos de Chile $\left(35^{\circ}-43^{\circ} 30^{\prime} \mathrm{S}\right)$
}

\author{
Reconstruction of vegetation cover and land use ca. 1550 and their change towards 2007 in the

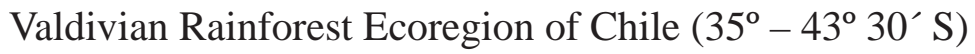

\author{
Antonio Lara ${ }^{\mathrm{a}, \mathrm{b} *}$, María Eugenia Solaric ${ }^{\mathrm{c}}$, María Del Rosario Prieto ${ }^{\mathrm{d}}$, María Paz Peña ${ }^{\mathrm{e}}$ \\ *Autor de correspondencia: ${ }^{a}$ Universidad Austral de Chile, Facultad de Ciencias Forestales y Recursos Naturales, \\ Instituto de Silvicultura, Casilla 567, Valdivia, Chile, tel.: 063-222151, antoniolara@uach.cl \\ ${ }^{\mathrm{b}}$ Fundación Centro de los Bosques Nativos FORECOS, Valdivia, Chile. \\ ${ }^{c}$ Universidad Austral de Chile, Facultad de Filosofía y Humanidades, Instituto de Ciencias Sociales, \\ Laboratorio de Arqueobotánica e Historia Ambiental, Valdivia, Chile.

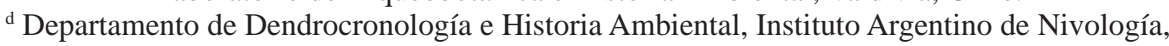 \\ Glaciología y Ciencias Ambientales (IANIGLA) CONICET, Mendoza, Argentina. \\ e Universidad Austral de Chile, Facultad de Ciencias, Instituto de Ciencias Ambientales y Evolutivas, Valdivia, Chile.
}

\begin{abstract}
SUMMARY
The estimation of the forest and vegetation cover in the Valdivian Rainforest Eco-region $\left(35^{\circ}-43^{\circ} 30^{\prime} \mathrm{S}\right)$ of Chile in the PreEuropean period emerges as a relevant scientific question due to its implications for forest conservation and for the improvement of the understanding of the patterns and dynamics of human settlement. This study uses a transdisciplinary approach integrating information from historical documents, archaeological evidence and the relationship of present vegetation with environmental variables. From this analysis we developed the first reconstruction of the forest and vegetation in the Valdivian Rainforest Eco-region circa 1550 (1:500.000 scale map). Our results indicate that native forests covered 11.3 million ha ca. 1550 at the Spanish conqueror arrival, decreasing to 5.7 million ha in 2007 (50.4\% of the original area). This reduction is due to the conversion of native forests to pasturelands, shrublands and agriculture land and, since 1974, forest plantations of exotic species. This study shows the need to continue developing transdisciplinary research, which integrates historical, archaeological and biophysical (potential vegetation, pollen analysis and dendrochronology) data to improve the understanding of changing forest and vegetation cover under climatic fluctuations and human influences during the last 450 years.
\end{abstract}

Key words: environmental history, native people, land use change, native forests, transdisciplinary studies.

\section{RESUMEN}

La estimación de la cobertura de vegetación y uso del suelo de la ecorregión de los bosques valdivianos lluviosos ( $35^{\circ}$ - $43^{\circ} 30^{`}$ S) en Chile antes de la colonización europea, surge como una interrogante relevante por sus implicancias para la conservación de la naturaleza y la comprensión de los patrones y dinámica del poblamiento humano. A partir de un enfoque transdisciplinario que integra información proveniente de documentos históricos, y sitios arqueológicos, y de la relación de estos registros así como de la vegetación actual con variables ambientales, se desarrolló la primera reconstrucción de la cobertura boscosa y de la vegetación de la ecorregión de los bosques valdivianos lluviosos en Chile hacia 1550, expresada cartográficamente a escala 1:500.000. Los resultados mostraron que, a la llegada de los españoles, los bosques nativos cubrían 11,3 millones de hectáreas, cifra que disminuyó en 2007 a 5,8 millones de hectáreas (51 \% del área original). Dicha reducción se explica por el reemplazo del bosque nativo por praderas y matorrales, áreas agrícolas y, a partir de 1974, por plantaciones forestales. Este estudio muestra la necesidad de continuar desarrollando una línea de investigación transdisciplinaria que integre métodos de investigación históricos, arqueológicos y biofísicos (vegetación potencial, palinología, dendrocronología), para lograr una mejor comprensión de los cambios ocurridos en la vegetación bajo fluctuaciones climáticas e influencias humanas durante los últimos 450 años.

Palabras clave: historia ambiental, pueblos originarios, bosque nativo, estudios transdisciplinarios.

\section{INTRODUCCIÓN}

La ecorregión de los bosques valdivianos lluviosos se desarrolla en Chile y áreas adyacentes de Argentina entre los paralelos $35^{\circ}$ y $48^{\circ} \mathrm{S}$, incluyendo varios tipos de bosques, matorrales, humedales, ríos y lagos (Dinerstein et al.
1995). Esta ecorregión ha sido clasificada entre aquellas con prioridad de conservación a nivel mundial, debido a su estatus de hot spot de biodiversidad, alto nivel de endemismos y rápida tasa de destrucción y degradación por causas antrópicas (Dinerstein et al. 1995, Myers et al. 2000, Echeverría et al. 2007). 
La estimación de la cobertura original de los bosques nativos hacia 1550 antes de la colonización europea en la zona sud-central de Chile ( $35^{\circ}-43^{\circ} 30^{\prime} \mathrm{S}$ ), cuando el territorio era ocupado por pueblos originarios, surge como una interrogante relevante. La estimación de la cobertura boscosa original tiene claras implicancias para el diseño de estrategias de conservación de los bosques de la ecorregión valdiviana. Las interpretaciones respecto a la vegetación boscosa existente en el centro-sur de Chile antes de la colonización europea han sufrido un cambio sustancial de enfoque en la última década. La interpretación original planteaba una población reducida dedicada a la caza, recolección y horticultura, con una escasa transformación antropogénica del paisaje (Latcham 1928, Elizalde 1968). Esta interpretación ha sido modificada drásticamente a partir de la relectura y análisis detallado de la documentación originada durante el siglo XVI (Bengoa 2003, Camus 2006, Otero 2006, Camus y Solari 2008), junto con las interpretaciones que se puedan extraer de la evidencia arqueológica y paleoambiental para el área de estudio. Estas evidencias muestran una sociedad agro-alfarera compleja y variada, que en el siglo XVI ocupaba los valles y riberas de ríos y lagos, señalando la presencia de extensas superficies de "tierras despejadas" para la agricultura y ganadería (Mariño de Lobera 1865, Vivar 1979, Rosales 1989). Por el contrario, los viajeros y exploradores que recorrieron la zona sur-central de Chile durante la segunda mitad del siglo XIX encontraron el territorio extensamente cubierto por bosques aparentemente no alterados, sin áreas despejadas (Darwin [1832] ${ }^{1}$ 1945, Treutler [1863] 1958, Philippi [1850] 2003). Este paisaje había resultado de la guerra de conquista (siglos XVII al XIX) que redujo la población original y llevó al abandono de tierras cultivables y praderas, desarrollando una nueva estrategia adaptativa basada en la ganadería trashumante de vacunos y ovinos introducidos por los europeos (Bengoa 2000, 2003, Guarda 2001, Torrejón y Cisternas 2002).

Dentro de este contexto, los objetivos de este trabajo son: a) reconstruir la cobertura de la vegetación entre los $35^{\circ}$ - $43^{\circ} 30 \mathrm{~S}$, hacia el año 1550, a partir de la integración de fuentes documentales y arqueológicas y su relación con variables ambientales, así como las relaciones entre vegetación actual y variables ambientales; b) determinar los cambios ocurridos en la cobertura de la vegetación y usos del suelo entre 1550 y 2007 y c) caracterizar los patrones de ocupación del territorio por parte de los pueblos originarios hacia 1550 y su efecto sobre la vegetación.

\section{MÉTODOS}

Área de estudio. La reconstrucción de la vegetación hacia 1550 se extiende entre los $35^{\circ}$ y $43^{\circ} 30^{\prime} \mathrm{S}$ abarcando cinco regiones administrativas desde la región del Maule a la

\footnotetext{
${ }^{1}$ En paréntesis cuadrado se indica la fecha original de publicación de cada trabajo.
}

de Los Lagos con un total de 16,6 millones de hectáreas. El área está caracterizada por tres unidades fisiográficas orientadas de Norte a Sur representadas por la Cordillera de la Costa, Depresión Intermedia y Cordillera de Los Andes (figura 1).

El clima en la porción norte del área de estudio $\left(35^{\circ} \mathrm{S}-\right.$ $38^{\circ} \mathrm{S}$ ) es de tipo templado mediterráneo con veranos secos (3 a 4 meses) y 700 a 1.200 mm de precipitación anual. Al sur de los $38^{\circ} \mathrm{S}$ el clima cambia al tipo templado lluvioso con precipitaciones anuales de 2.000 a $4.000 \mathrm{~mm}$ (Miller 1976). Además, hay un gradiente Oeste-Este, donde las precipitaciones disminuyen hacia la Depresión Intermedia debido al efecto de sombra de lluvia causado por la Cordillera de la Costa, y vuelven a aumentar al aproximarse a la Cordillera de Los Andes (figura 1, Miller 1976).

Los bosques nativos en el área de estudio tienen una composición muy variada, siguiendo el gradiente bioclimático de norte a sur. Actualmente, en el área norte los bosques naturales corresponden a formaciones abiertas del tipo esclerófilo (Donoso 1993). Hacia los $37^{\circ} \mathrm{S}$ dominan los bosques caducifolios del género Nothofagus. En la porción sur del área de estudio prevalecen los bosques latifoliados perennifolios formados por una mezcla de especies, clasificados como bosques del tipo forestal siempreverde (valdiviano). A mayor altitud y hasta el límite de la vegetación arbóreo dominan los bosques del tipo forestal lenga (Donoso 1993).

Categorías de clasificación de la vegetación y uso del suelo. Dicha clasificación que integra el tipo de vegetación y forma en que es usado el suelo, siguió el esquema desarrollado por CONAF et al. (1999), descrito por Lara y Sandoval (2003). Para el año 2007 se identificaron las categorías: bosque nativo, praderas y matorrales, áreas agrícolas, plantaciones forestales, áreas urbanas y otros usos. Para el período hacia 1550, sólo se incluyó bosque nativo, praderas y matorrales, mosaico y otros usos.

- Bosque nativo. Áreas en que los árboles nativos tienen una cobertura de copas $\geq 25 \%$. Incluye bosques adultos, renovales (bosques jóvenes secundarios), adultorenoval y bosques achaparrados.

- Praderas y matorrales. Formaciones donde los arbustos y las especies herbáceas, juntos o por separado, cubren al menos el 25 \% del área.

- Mosaico. Definida a partir de la descripción e interpretación histórica de los patrones de ocupación del territorio de los pueblos originarios (siglos XVI-XVIII). Categoría formada por parches de tamaño variable dominados por praderas, matorrales, áreas agrícolas, y asentamientos humanos resultantes de la eliminación del bosque. No obstante estar dominada por áreas despejadas, incluye rodales de bosque.

- Plantaciones forestales. Áreas dominadas por plantaciones mayoritariamente de Pinus radiata D. Don, y Eucalyptus spp. para abastecer la industria forestal y en menor medida para leña. 


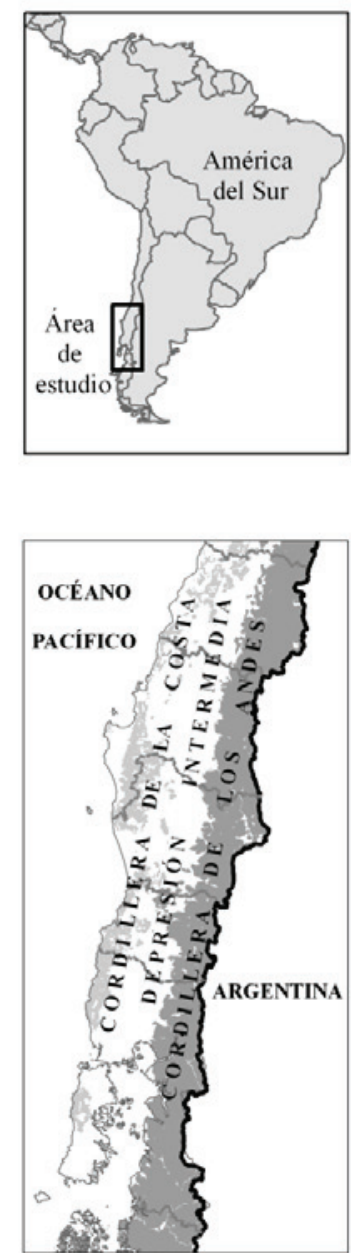

Leyenda

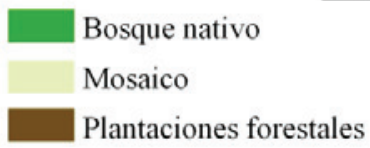

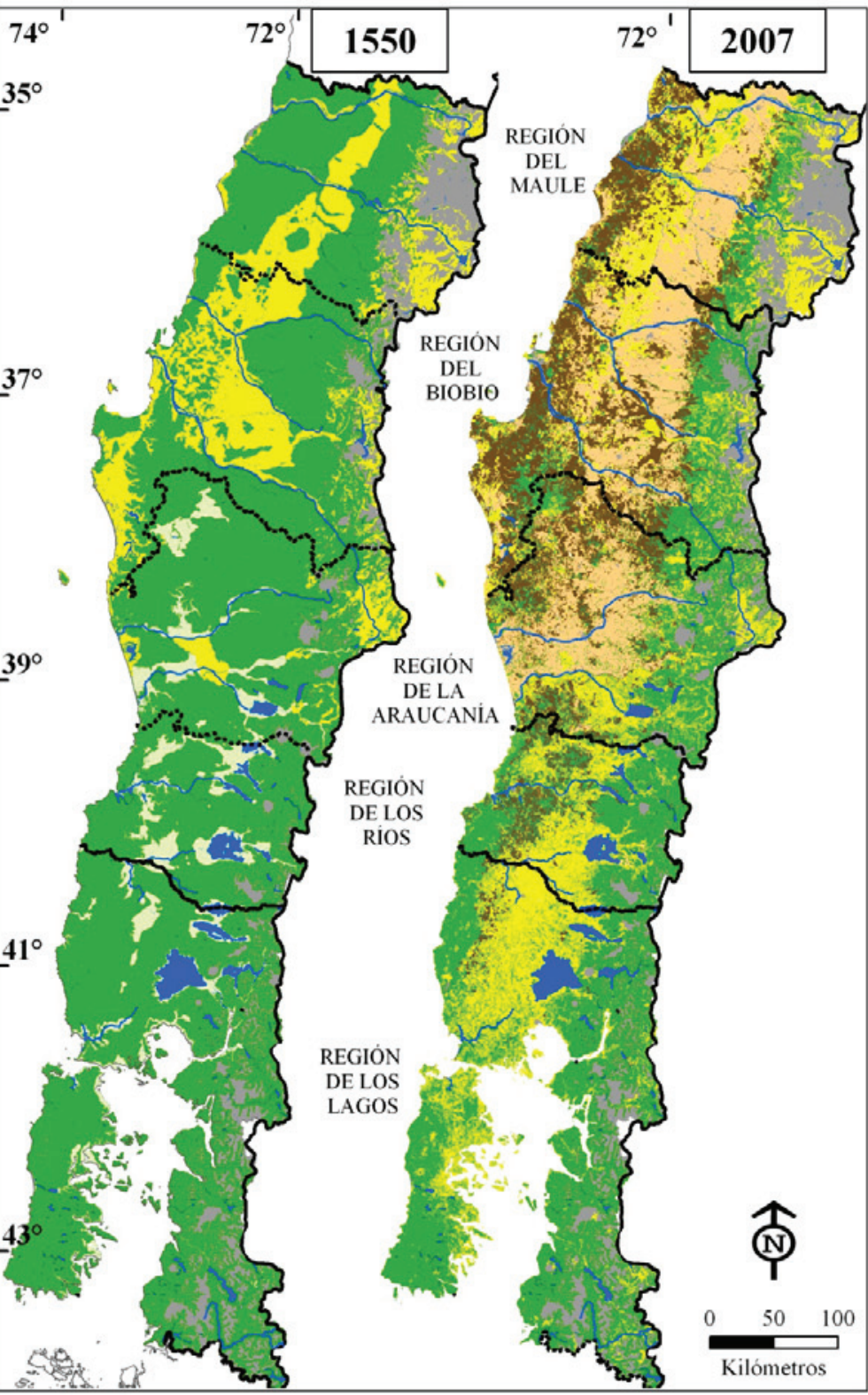

Praderas y matorrales _ Sin vegetación, nieves y glaciares Áreas agricolas

Lagos y ríos principales ........ Limites regionales

Figura 1. Ubicación del área de estudio, cobertura de la vegetación y de uso del suelo en la ecorregión valdiviana ( $\left.35^{\circ} \mathrm{S}-43^{\circ} 30^{\prime} \mathrm{S}\right)$ hacia 1550 y en 2007. Las categorías áreas urbanas y humedales no son mostradas.

Location of the study area, and vegetation - land use cover in the Valdivian Rainforest Ecoregion (35 $\left.\mathrm{S}-43^{\circ} 30^{\prime} \mathrm{S}\right) \mathrm{ca} .1550$ and in 2007. Urban areas and wetlands are not shown.

- Áreas agrícolas. Incluye todo tipo de cultivos anuales y permanentes.

- Áreas urbanas. Zonas ocupadas por pueblos y ciudades.

- Lagos y ríos principales, los cuales sólo se muestran en el mapa de cobertura vegetacional (figura 1). Para el cálculo de áreas, se incluyen en la categoría otros usos.

- Otros usos del suelo. Incluye: áreas desprovistas de vegetación, nieves y glaciares, humedales y áreas no reconocidas.

Generación de un mapa sinóptico de la cobertura boscosa en 2007. Se utilizaron como base las coberturas digitales en ArcView 3.1® de los mapas de vegetación natural de Chile realizados por CONAF et al. (1999) y sus actuali- 
zaciones a 2007 en ArcGis 9.3® para las diferentes regiones administrativas (CONAF et al. 2000, 2008ab, 2009). A partir de estas coberturas, a una escala 1:50.000 con un tamaño mínimo de polígono de 6,25 ha, obtenidos de la interpretación de fotografías aéreas y de descripciones de terreno se desarrolló un mapa a una escala 1:500.000 con las categorías definidas anteriormente.

Desarrollo del mapa de cobertura de vegetación y usos del suelo hacia 1550. La elaboración de este mapa se realizó en las siguientes etapas: 1) búsqueda y sistematización de información contenida en documentos históricos escritos por cronistas, misioneros y viajeros que visitaron el área de estudio a partir de la segunda mitad del siglo XVI, los cuales hacían referencia a asentamientos humanos, menciones al paisaje y la vegetación (por ejemplo áreas cubiertas por cultivos y praderas versus áreas boscosas); 2) ubicación y delimitación de estas descripciones usando como referencia accidentes geográficos, localidades y su incorporación a un sistema de información geográfica (SIG), utilizando ArcGis 9.3® con un nivel de detalle correspondiente a una escala $1: 500.000 ; 3)$ recopilación de registros arqueológicos en las zonas donde existe una sistematización de estos, los cuales fueron ubicados geográficamente e incorporados al SIG, aportando información única o complementaria para delimitar las áreas ocupadas por praderas, matorrales y mosaico; 4) estimación de la vegetación para las áreas sin información histórica o arqueológica. Esto último fue hecho de dos formas complementarias. La primera, a partir del análisis de las relaciones entre los asentamientos humanos documentados y variables ambientales, incluyendo unidad fisiográfica, relieve, rango de altitud, drenaje de los suelos y su aptitud agrícola, así como distancia a los ríos, lagos y borde costero. De este modo, por ejemplo, las áreas de mosaico aledañas a ríos y lagos definidas por registros históricos se extrapolaron a otras zonas de similares características ambientales. La segunda forma de estimación de la vegetación original para áreas sin registros históricos o arqueológicos y en que no se había inferido la presencia de praderas, matorrales o mosaicos se basó en la relación entre la vegetación actual y las variables ambientales. Por ejemplo, el rango altitudinal o tipo de drenaje en que se presentan los bosques nativos en la actualidad en una región determinada, fueron utilizados para delimitar este tipo de uso de suelo en áreas actualmente cubiertas por praderas y matorrales o plantaciones que se desarrollan en esos rangos ambientales. Para las áreas actualmente cubiertas de bosque nativo en que no se tienen registros o no se infirió la ocupación o intervención por parte de los pueblos originarios, se asumió que la cobertura original era bosque nativo.

En el caso de los documentos históricos, se utilizó el análisis de contenido (Prieto et al. 2003) para la extracción y categorización de la información, reduciendo la cantidad de expresiones producida por cronistas, militares, religiosos, viajeros e historiadores $y$, de este modo, obtener datos más homogéneos. Además se usó el método fisonómico fundamentado en la identificación de grandes unidades de vegetación (bosques, matorrales, praderas) tal como se presentan al observador. Se utilizó principalmente la documentación de mediados del siglo XVI al siglo XVIII, considerando este último período sólo para las áreas periféricas al poblamiento indígena original.

La recopilación de antecedentes históricos incluyó más de 350 referencias documentales, que permitieron ubicar un total de aproximadamente 900 puntos o áreas sobre las cuales se obtuvo una descripción histórica de la cobertura de la vegetación. Para el análisis de la información de sitios arqueológicos que señalaban presencia de asentamientos humanos se usó sólo estudios sistemáticos realizados principalmente en las regiones de La Araucanía y de Los Ríos (Aldunate 1989, Dillehay 1990, 2003, 2007, Navarro 2004, Adán et al. 2007). Se estima que las áreas delimitadas en el mapa de vegetación de 1550, para las cuales se contaba con información de documentos históricos o estudios arqueológicos, corresponden a un 10 a $15 \%$ de las áreas clasificadas como mosaico, praderas y matorrales. Este porcentaje no incluye las praderas y matorrales del sector alto-andino.

La estimación de la cobertura de la vegetación hacia 1550 no consideró la existencia de cambios ocurridos en la distribución actual de áreas desprovistas de vegetación sobre el límite altitudinal de los bosques, así como de las categorías nieves y glaciares, humedales, lagos y ríos principales, respecto al año 2007. Tampoco se consideraron las posibles modificaciones en la cobertura boscosa y de la vegetación debido a cambios climáticos o retroceso de glaciares.

Obtención de la superficie de cambio. Utilizando el software ArcGis 9.3 \& se realizaron cruces entre las coberturas de vegetación en el año 2007 y hacia 1550, ambas a escala 1:500.000, para cada una de las regiones administrativas, generándose una matriz de cambio entre las diferentes categorías, y posteriormente se estimó el área de dichos cambios. Se reconoce que el cambio de la cobertura de la vegetación es dinámica a lo largo del tiempo, pero esta variabilidad no fue analizada en este estudio.

\section{RESULTADOS}

Cobertura de la vegetación hacia el año 1550. El bosque nativo dominaba ampliamente el paisaje entre los $35^{\circ} \mathrm{y}$ $43^{\circ} 30^{\prime} \mathrm{S}$, cubriendo 11,3 millones de hectáreas, lo que representa un 68 \% del área de estudio (figura 1, cuadro 1). Además, se observa un aumento de la importancia relativa del bosque nativo de Norte a Sur siguiendo un gradiente climático hacia áreas de mayor precipitación. De esta manera, en la región del Maule el bosque nativo estimado hacia 1550 representaba un 55 \%, mientras que en las regiones de La Araucanía, Los Ríos y Los Lagos representaba entre un $74 \%$ y $77 \%$ del área total (cuadro 2). Por otro lado, en la porción norte del área de estudio (regiones 
Cuadro 1. Área (hectáreas) de las diferentes categorías de vegetación y uso del suelo hacia 1550 y 2007 en el área de estudio.

Area (hectares) of each vegetation - land use category in the study area for $c a .1550$ and 2007.

\begin{tabular}{lrrrr}
\hline & \multicolumn{5}{c}{ Año } \\
\cline { 2 - 5 } Uso del suelo & \multicolumn{2}{c}{ Hacia 1550} & \multicolumn{1}{c}{2007} \\
\cline { 2 - 5 } & \multicolumn{1}{c}{ ha } & \multicolumn{1}{c}{ ha } & \% \\
\hline Bosque nativo & 11.335 .239 & 68,3 & 5.762 .640 & 34,7 \\
Praderas y & 2.283 .605 & 13,8 & 3.776 .615 & 22,7 \\
matorrales & 890.928 & 5,4 & - & - \\
Mosaico & - & - & 2.521 .316 & 15,2 \\
Áreas agrícolas & & - & 2.383 .990 & 14,4 \\
Plantaciones & - & - & 65.211 & 0,4 \\
forestales & - & - & 2.096 .811 & 12,6 \\
Áreas urbanas & 2.096 .811 & 12,6 & 16.606 .583 & 100,0 \\
Otros usos a & 16.606 .583 & 100,0 & \\
Total & & & &
\end{tabular}

${ }^{a}$ Incluye: áreas desprovistas de vegetación, nieves y glaciares, lagos, ríos principales, humedales y áreas no reconocidas.

de Maule y Biobío) los bosques estaban concentrados en las cordilleras de la Costa y de Los Andes, siendo escasos en la Depresión Intermedia (figura 1). Ello, a diferencia de una distribución más amplia y homogénea en las regiones de La Araucanía, Los Ríos y Los Lagos (figura 1).

La segunda categoría en importancia que dominaba la vegetación hacia 1550 eran las praderas y matorrales que cubrían un total de 2,3 millones de hectáreas (14 \% del total, cuadro 1), siendo especialmente relevantes en las regiones del Maule y Biobío, donde representaban un $25 \%$ y $33 \%$ del total regional, respectivamente, concentrándose en la Depresión Intermedia, áreas costeras y ribereñas (figura 1). La categoría mosaico abarcaba un total de 891 mil hectáreas (5 \% del total), alcanzando su máxima importancia relativa en las regiones de La Araucanía y Los Ríos donde cubría un 10 \% y 15 \% del área total regional, respectivamente (cuadro 2). Los mosaicos se distribuían principalmente en los valles de la vertiente oriental de la Cordillera de la Costa, en la Depresión Intermedia, a lo largo de los ríos y en torno a los lagos principales (figura 1).

Cambio de la cobertura de la vegetación y uso de suelo entre 1550 y 2007. Entre las regiones del Maule y de La Araucanía uno de los principales cambios fue el desarrollo de áreas agrícolas en la Depresión Intermedia, que abarcaron entre $23 \%$ y $27 \%$ de los totales regionales en el año 2007 (figura 1, cuadro 2). Otro cambio importante fue la expansión de las plantaciones forestales en las regiones del Maule, Biobío y Araucanía, donde estas representan en 2007 un 17 \%, 28 \% y 19 \% del área total, respectivamente (figura 1, cuadro 2).

Por otro lado, la cobertura de bosque nativo se ha reducido desde 11,3 millones de hectáreas a 5,8 millones de hectáreas entre 1550 y 2007. Esta última cifra representa un $51 \%$ de la cobertura boscosa original (cuadro 1). La re- gión del Maule presenta la mayor disminución porcentual de bosque nativo y, en 2007, sólo permanecía un $22 \%$ de la cobertura boscosa original (figura 2). Las regiones de Los Ríos y Los Lagos son las que han mantenido el mayor porcentaje de bosques remanentes con un $66 \%$ y $74 \%$ de la cobertura hacia 1550 , respectivamente (figura 2 ).

El cambio de bosque nativo a otros usos del suelo para las diferentes regiones muestra que en Maule y Biobío las áreas cubiertas originalmente por bosques nativos están actualmente ocupadas por plantaciones forestales (29\% para ambas regiones), áreas agrícolas (22 \% y $25 \%$ ), y praderas y matorrales (28 \% y $12 \%$, respectivamente) (cuadro 3, figura 3A). Estos cambios han ocurrido principalmente en la Depresión Intermedia y en la Cordillera de la Costa, de tal manera que los bosques nativos remanentes se concentran fuertemente en el sector andino. En La Araucanía, los mayores porcentajes de cambio de los bosques nativos corresponden a áreas agrícolas (26\%), seguidas de plantaciones forestales (22\%) y de praderas y matorrales (15\%) (cuadro 3, figura 3A). En las regiones de Los Ríos y Los Lagos el bosque nativo ha sido reemplazado principalmente por praderas y matorrales ( $25 \% \mathrm{y}$ $27 \%$, respectivamente) (cuadro 3, figura 3A).

Otras categorías de cobertura de la vegetación que experimentaron cambios importantes son las praderas y ma-

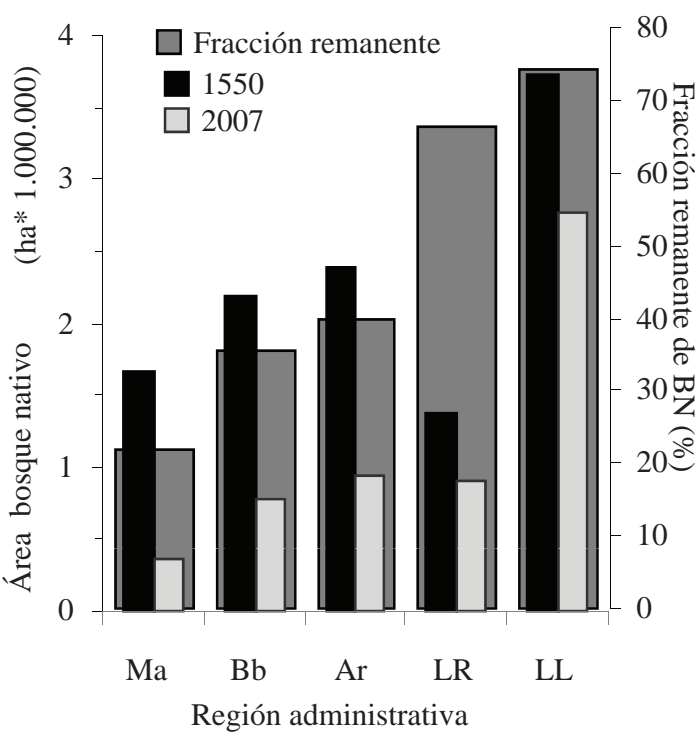

Figura 2. Área de bosque nativo años 1550 y 2007 (en millones de hectáreas) y fracción remanente (\%) respecto a 1550 para cada región. La fracción remanente fue calculada como: [(Área en 2007 / Área original hacia 1550) * 100]. Ma: Maule, Bb: Biobío, Ar: Araucanía, LR: Los Ríos, LL: Los Lagos, BN: bosque nativo, ha: hectáreas.

Native forest area ca. 1550 and in 2007 (hectares x 1000) and remnant percentage (\%) relative to $\mathrm{ca}$. 1550 for each region. The remnant percentage was calculated as [(Area in 2007/Area ca. 1550) * 100]. Ma: Maule, Bb: Biobío, Ar: Araucanía, LR: Los Ríos, LL: Los Lagos, BN: bosque nativo. 
Cuadro 2. Área (hectáreas) de las diferentes categorías de vegetación y uso de suelo para cada región hacia 1550 y 2007. Area (hectares) of each vegetation - land use category for each region ca. 1550 and 2007.

\begin{tabular}{|c|c|c|c|c|c|}
\hline \multirow{3}{*}{ Región } & \multirow{3}{*}{ Vegetación y uso de suelo } & \multicolumn{4}{|c|}{ Año } \\
\hline & & \multicolumn{2}{|c|}{ Hacia 1550} & \multicolumn{2}{|l|}{2007} \\
\hline & & hectáreas & $\%$ & hectáreas & $\%$ \\
\hline \multirow[t]{8}{*}{ Maule } & Bosque nativo & 1.665 .293 & 54,9 & 363.961 & 12,0 \\
\hline & Praderas y matorrales & 770.008 & 25,4 & 846.768 & 27,9 \\
\hline & Mosaico & - & - & - & - \\
\hline & Áreas agrícolas & - & - & 696.324 & 22,9 \\
\hline & Plantaciones forestales & - & - & 516.026 & 17,0 \\
\hline & Áreas urbanas & - & - & 12.222 & 0,4 \\
\hline & Otros usos ${ }^{\mathrm{a}}$ & 600.292 & 19,8 & 600.292 & 19,8 \\
\hline & Total regional & 3.035 .593 & 100,0 & 3.035 .593 & 100,0 \\
\hline \multirow[t]{8}{*}{ Biobío } & Bosque nativo & 2.187 .029 & 59,0 & 777.264 & 21,0 \\
\hline & Praderas y matorrales & 1.224 .223 & 33,0 & 602.473 & 16,2 \\
\hline & Mosaico & - & - & - & - \\
\hline & Áreas agrícolas & - & - & 984.907 & 26,6 \\
\hline & Plantaciones forestales & - & - & 1.021 .639 & 27,5 \\
\hline & Áreas urbanas & - & - & 24.969 & 0,7 \\
\hline & Otros usos ${ }^{\text {a }}$ & 297.413 & 8,0 & 297.413 & 8,0 \\
\hline & Total regional & 3.708 .665 & 100,0 & 3.708 .665 & 100,0 \\
\hline \multirow[t]{8}{*}{ Araucanía } & Bosque nativo & 2.383 .976 & 74,9 & 947.943 & 29,8 \\
\hline & Praderas y matorrales & 289.374 & 9,1 & 611.626 & 19,2 \\
\hline & Mosaico & 316.507 & 9,9 & - & - \\
\hline & Áreas agrícolas & - & - & 815.732 & 25,6 \\
\hline & Plantaciones forestales & - & - & 600.890 & 18,9 \\
\hline & Áreas urbanas & - & - & 13.666 & 0,4 \\
\hline & Otros usos ${ }^{a}$ & 191.567 & 6,0 & 191.567 & 6,0 \\
\hline & Total regional & 3.181 .424 & 100,0 & 3.181 .424 & 100,0 \\
\hline \multirow[t]{8}{*}{ Los Ríos } & Bosque nativo & 1.367 .905 & 74,3 & 907.612 & 49,3 \\
\hline & Praderas y matorrales & - & - & 532.007 & 28,9 \\
\hline & Mosaico & 284.109 & 15,4 & - & - \\
\hline & Áreas agrícolas & - & - & 16.275 & 0,9 \\
\hline & Plantaciones forestales & - & - & 190.374 & 10,3 \\
\hline & Áreas urbanas & - & - & 5.746 & 0,3 \\
\hline & Otros usos ${ }^{a}$ & 188.299 & 10,2 & 188.299 & 10,2 \\
\hline & Total regional & 1.840 .313 & 100,0 & 1.840 .313 & 100,0 \\
\hline \multirow[t]{8}{*}{ Los Lagos } & Bosque nativo & 3.731 .036 & 77,1 & 2.765 .860 & 57,1 \\
\hline & Praderas y matorrales & - & - & 1.183 .741 & 24,5 \\
\hline & Mosaico & 290.312 & 6,0 & - & - \\
\hline & Áreas agrícolas & - & - & 8.078 & 0,17 \\
\hline & Plantaciones forestales & - & - & 55.061 & 1,1 \\
\hline & Áreas urbanas & - & - & 8.608 & 0,2 \\
\hline & Otros usos ${ }^{\text {a }}$ & 819.240 & 16,9 & 819.240 & 16,9 \\
\hline & Total regional & 4.840 .588 & 100,0 & 4.840 .588 & 100,0 \\
\hline
\end{tabular}

a Incluye: áreas desprovistas de vegetación, nieves y glaciares, lagos, ríos, humedales y áreas no reconocidas.

torrales, y mosaico. El principal cambio que experimentaron las praderas y matorrales fue su reemplazo por áreas agrícolas (834 mil hectáreas), seguido por plantaciones forestales (435 mil hectáreas) y, en menor medida, por la recuperación de bosque nativo (99 mil hectáreas) (cuadro 3). A nivel regional, es en Maule y Biobío donde ocurre el mayor cambio a áreas agrícolas ( $42 \%$ y $36 \%$ del total original de praderas y matorrales, figura 3B). Sólo en la región del Biobío destaca el cambio a plantaciones forestales con un $32 \%$ del total original (figura 3B). Por otro lado, las áreas mosaicos presentaron cambios principalmente a praderas y matorrales (454 mil hectáreas), y en segundo lugar a bosque nativo (186 mil hectáreas) y a áreas agrícolas (142 mil hectáreas) (cuadro 3). En las regiones de Los Ríos y Los Lagos un 65 \% del total original de mosaico cambió a praderas y matorrales (figura 3C). Para estas regiones, se destaca un cambio de mosaico a bosque nativo de un $21 \%$ y $31 \%$, respectivamente (figura $3 \mathrm{C}$ ). 
Cuadro 3. Cambios en el área (hectáreas) cubierta por bosque nativo, praderas y matorrales, y mosaico hacia 1550 a otras categorías de vegetación y uso de suelo en 2007.

Changes in the area (hectares) covered by native forests, grasslands and shrublands and mosaic of forest - open areas ca. 1550 to other vegetation - land use categories in 2007.

\begin{tabular}{|c|c|c|c|c|c|c|c|}
\hline \multirow[b]{2}{*}{ Región } & \multirow{2}{*}{$\begin{array}{l}\text { Vegetación y usos de } \\
\text { suelo en } 1550\end{array}$} & \multicolumn{5}{|c|}{ Vegetación y usos de suelo en 2007} & \multirow[b]{2}{*}{ Total en 1550} \\
\hline & & $\begin{array}{c}\text { Bosque } \\
\text { nativo }\end{array}$ & $\begin{array}{l}\text { Praderas y } \\
\text { matorrales }\end{array}$ & $\begin{array}{c}\text { Áreas } \\
\text { agrícolas }\end{array}$ & $\begin{array}{l}\text { Plantaciones } \\
\text { forestales }\end{array}$ & $\begin{array}{l}\text { Áreas } \\
\text { urbanas }\end{array}$ & \\
\hline \multirow{3}{*}{ Del Maule } & Bosque nativo & 347.437 & 459.332 & 370.343 & 484.257 & 3.924 & 1.665 .293 \\
\hline & Praderas y matorrales & 16.524 & 387.436 & 325.981 & 31.769 & 8.298 & 770.008 \\
\hline & Total en 2007 & 363.961 & 846.768 & 696.324 & 516.026 & 12.222 & - \\
\hline \multirow{3}{*}{ Del Biobío } & Bosque nativo & 737.804 & 265.252 & 543.947 & 630.394 & 9.632 & 2.187.029 \\
\hline & Praderas y matorrales & 39.460 & 337.221 & 440.960 & 391.245 & 15.337 & 1.224 .223 \\
\hline & Total en 2007 & 777.264 & 602.473 & 984.907 & 1.021 .639 & 24.969 & - \\
\hline \multirow{4}{*}{ La Araucanía } & Bosque nativo & 872.254 & 363.774 & 617.737 & 524.947 & 5.264 & 2.383.976 \\
\hline & Praderas y matorrales & 42.572 & 166.550 & 67.063 & 12.207 & 982 & 289.374 \\
\hline & Mosaico & 33.117 & 81.302 & 130.932 & 63.736 & 7.420 & 316.507 \\
\hline & Total en 2007 & 947.943 & 611.626 & 815.732 & 600.890 & 13.666 & - \\
\hline \multirow{3}{*}{ Los Ríos } & Bosque nativo & 845.882 & 348.769 & 7.523 & 164.386 & 1.345 & 1.367 .905 \\
\hline & Mosaico & 61.730 & 183.238 & 8.752 & 25.988 & 4.401 & 284.109 \\
\hline & Total en 2007 & 907.612 & 532.007 & 16.275 & 190.374 & 5.746 & - \\
\hline \multirow{3}{*}{ Los Lagos } & Bosque nativo & 2.675 .056 & 994.396 & 5.401 & 51.521 & 4.662 & 3.731 .036 \\
\hline & Mosaico & 90.804 & 189.345 & 2.677 & 3.540 & 3.946 & 290.312 \\
\hline & Total en 2007 & 2.765 .860 & 1.183 .741 & 8.078 & 55.061 & 8.608 & - \\
\hline \multirow{4}{*}{$\begin{array}{l}\text { Total área de } \\
\text { estudio }\end{array}$} & Bosque nativo & 5.478 .433 & 2.431 .523 & 1.544 .951 & 1.855 .505 & 24.827 & 11.335.239 \\
\hline & Praderas y matorrales & 98.556 & 891.207 & 834.004 & 435.221 & 24.617 & 2.283 .605 \\
\hline & Mosaico & 185.651 & 453.885 & 142.361 & 93.264 & 15.767 & 890.928 \\
\hline & Total en 2007 & 5.762 .640 & 3.776 .615 & 2.521 .316 & 2.383 .990 & 65.211 & - \\
\hline
\end{tabular}

\section{DISCUSIÓN}

Debido a la relevancia de la ecorregión de los bosques valdivianos lluviosos para la conservación a nivel global, se requiere estimar la cobertura de los bosques y los diferentes usos del suelo hacia 1550, antes de la colonización española, así como su comparación con la cobertura actual. La reconstrucción de la vegetación otorga la oportunidad de entender de mejor manera los patrones indígenas de ocupación del territorio y de los procesos que han llevado a los cambios en la cobertura vegetacional. También permite determinar las fracciones remanentes de bosques nativos protegidos actualmente en las áreas silvestres respecto al área que ocupaban dichos bosques hacia 1550 .

Patrones indígenas de ocupación del territorio hacia 1550. La eliminación del bosque nativo para desarrollar una agricultura y ganadería de pequeña y mediana escala por los pueblos originarios ha sido descrita en estudios arqueológicos y documentos históricos. Ello es concordante con evidencias encontradas en perfiles de polen y en estudios de estructura de bosques e historia de incen- dios registrados en los anillos de crecimiento (Veblen et al. 1979, Lara et al. 1999, González et al. 2005, Dillehay 2007, Abarzúa 2009). Posteriormente ocurrió un importante cambio del paisaje, desde uno con importantes áreas despejadas en que se asentaba la población originaria en torno a 1550, hacia uno con una mayor cobertura de bosques. Lo anterior, debido al abandono de los territorios mapuche por los continuos conflictos y guerras con los españoles, desde fines del siglo XVI hasta el siglo XIX, lo que permitió el establecimiento de bosques nativos en terrenos de cultivo y praderas abandonadas (Darwin [1832] 1945, Treutler [1863] 1958, Donoso 1983, Philippi [1850] 2003). Durante este período ocurrió un proceso de reducción poblacional, a la vez que un cambio en la estrategia adaptativa en que los mapuches adoptan la ganadería ovina y bovina europea, que representa una estrategia que les da mayor movilidad.

Un análisis regional muestra que en el Maule, el clima mediterráneo favoreció el asentamiento de los pueblos de tradición histórica picunche, principalmente en amplias áreas de la Depresión Intermedia, lo cual determinó que esta estuviera dominada por praderas y matorrales (figura 1) y terrenos agrícolas (estos últimos no delimitados en 


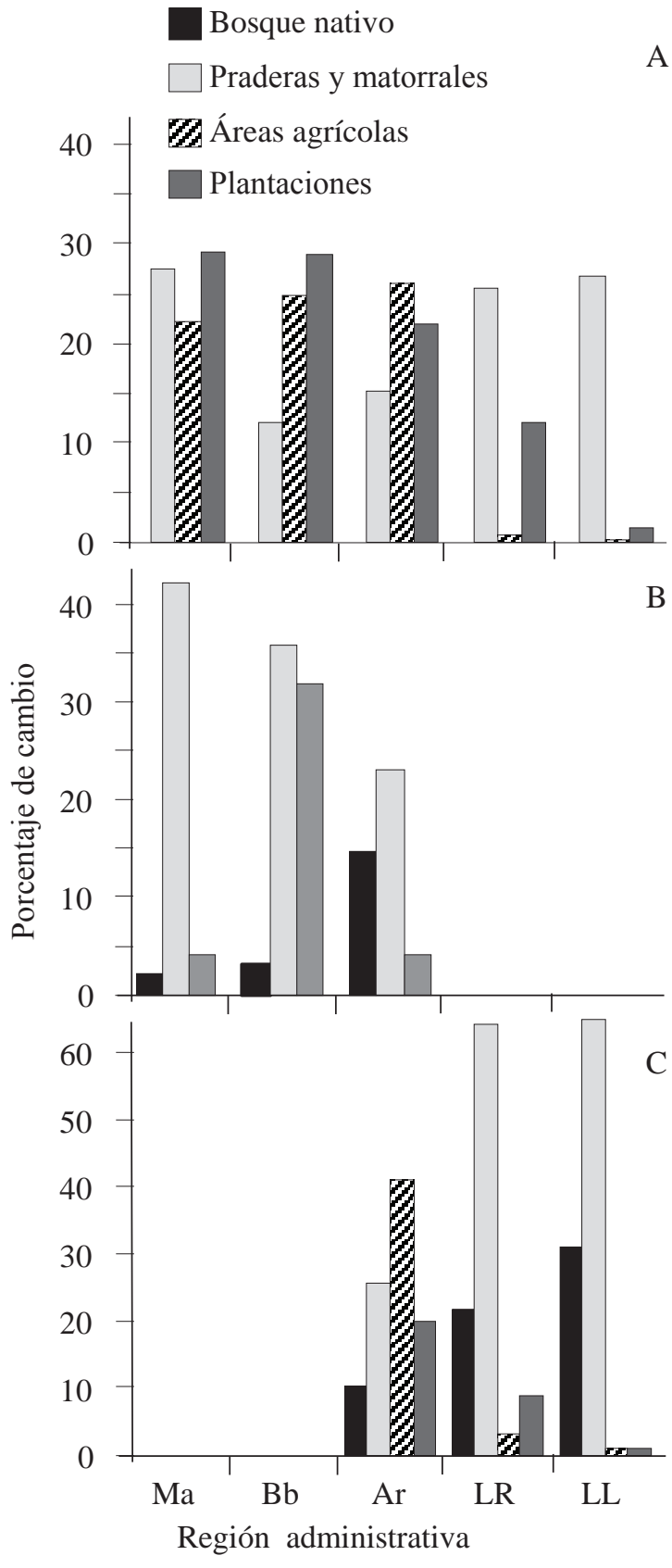

Figura 3. Cambios de uso del suelo y vegetación, en cada región administrativa, ocurridos entre ca. 1550 y 2007 desde (A): bosque nativo, (B): praderas y (C): mosaico. Los porcentajes están calculados con respecto al área original de cada categoría de vegetación y uso de suelo hacia 1550. Ma: Maule, Bb: Biobío, Ar: Araucanía, LR: Los Ríos, LL: Los Lagos. La figura no muestra el porcentaje del área de cada categoría que permaneció sin cambios.

Change from native forest (A), shrublands and grasslands (B), and mosaic of forest - open areas (C) ca. 1550 to other vegetation land use cover categories in 2007 for each region. Percentages calculated respect to the original area for each category $c a$. 1550. The percentages of each category without changes are not shown. Ma: Maule, Bb: Biobío, Ar: Araucanía, LR: Los Ríos, LL: Los Lagos. el mapa debido a la ausencia de información suficiente). La menor capacidad de recuperación ante las quemas y desmonte, debido a las limitaciones climáticas y de fertilidad de suelo, explicaría la permanencia de una cobertura de arbustos y pastos hasta la actualidad. El bosque nativo estaba restringido principalmente a la precordillera andina (Sanhueza et al. 1994).

Durante el siglo XVI la región del Biobío poseía amplias áreas de praderas y matorrales en la Depresión Intermedia (figura 1). Este patrón de ocupación se asocia al desarrollo de asentamientos humanos en terrenos fértiles asociados a una agricultura de secano y a una ganadería incipiente del chiliweke (camélido relacionado al guanaco) (Mariño de Lovera 1865, Vivar 1979). También se observan importantes zonas despejadas de bosque en el sector costero que obedecía a asentamientos asociados a la disponibilidad de importantes recursos marinos (figura 1) (Aldunate 1989, Navarro 2004).

La presencia de praderas y mosaicos en las regiones de La Araucanía y de Los Ríos hacia 1550 (figura 1) es consistente con recientes estudios arqueológicos que demuestran una importante complejización socio-política desde siglo XIII hasta el XVI con la agricultura en valles, como Purén y Lumaco y en las riberas de ríos y lagos, principales vías de comunicación e intercambio (Aldunate 1989, Bengoa 2003, Dillehay 2003, 2007). Esto es confirmado por estudios paleoambientales realizados en Purén y Lumaco ( $\left.38^{\circ} \mathrm{S}\right)$ y en el lago Budi $\left(39^{\circ} \mathrm{S}\right)$, que describen la presencia de polen de maíz (Zea mays L.) a partir de $\sim 5$ ka AP (Abarzúa 2009). Las áreas de mosaico en la región de Los Lagos siguen un patrón similar, estando asociadas a valles, riberas de ríos y lagos en que se asentaba la población de tradición mapuche. Los patrones de ocupación descritos se ven claramente representados en la figura 1.

Estudios basados en la estructura de los bosques evidencian los asentamientos mapuches en zonas lacustres. En la ribera norte del lago Villarrica, Veblen et al. (1979) describen un bosque dominado por una cohorte coetánea de Nothofagus obliqua (Mirb) Oerst., que se estableció en condiciones abiertas hace al menos 250 años producto de un incendio causado por la población originaria como parte de una estrategia agrícola-hortícola. Investigaciones basadas en anillos de crecimiento documentan la ocurrencia de incendios reiterados en bosques de araucaria en el territorio original de los pehuenches, desde 1450 (González et al. 2005) y en bosques de alerce en la Cordillera de la Costa (40 S) desde el año 1397 (Lara et al. 1999), causados por rayos o por los habitantes originarios.

El área total de mosaico, praderas y matorrales donde se asentó la población originaria, según los patrones descritos y las estimaciones de población en la segunda mitad del siglo XVI, parecen consistentes. Diversos antropólogos e historiadores proponen esta población en torno a un millón de personas entre el río Maule y Chiloé (Bengoa 2000, Tellez 2004). El presente estudio estima un área de 3,2 millones de hectáreas de mosaicos, praderas y mato- 
rrales hacia 1550, lo que señalaría una densidad poblacional de 0,31 habitantes por hectárea. La estimación parece adecuada en relación al tipo de organización económica y política de los mapuches, cuyos asentamientos correspondían a una población sedentaria, agro-ganadera, en la cual la caza, la pesca y la recolección eran complementarias (Dillehay 1990).

Procesos de cambio en la vegetación y uso del suelo entre 1550 y 2007. Los principales cambios en la cobertura de la vegetación en el período son la drástica disminución de la superficie cubierta por bosque nativo, junto al aumento de las praderas y matorrales y la aparición de nuevos usos como agricultura de gran escala y plantaciones forestales (figura 1, cuadros 1 y 2).

La transformación del paisaje ha sido de tal magnitud que, en la actualidad, la cobertura de bosque nativo es aproximadamente la mitad de la existente hacia 1550, aumentando además el grado de fragmentación de esta categoría (figura 1, cuadro 1). El principal cambio de bosques nativos hacia otros usos del suelo ha sido a praderas y matorrales, plantaciones forestales y áreas agrícolas, representando un $21 \%, 16 \%$ y $14 \%$ de la superficie de cambio, respectivamente (cuadro 3). Dentro de esta tendencia general hacia el reemplazo de los bosques nativos por otros usos del suelo, es interesante destacar que un total de 284 mil hectáreas de mosaico, praderas y matorrales que existían hacia 1550 , se transformaron a bosque nativo en 2007 (cuadro 3, figura 3).

El proceso de reemplazo de bosques nativos para la expansión de la agricultura y ganadería, especialmente en la Depresión Intermedia, se inició con la colonización europea en las regiones de La Araucanía a Los Lagos a partir de 1850, ocasionando la masiva destrucción y fragmentación de estos bosques (Camus 2006, Otero 2006, Armesto et al. 2010). Este proceso de destrucción del bosque nativo continuó durante el siglo XX y hasta la década de 1960, debido a grandes incendios, y habilitación de praderas, impulsados por leyes que promovían la colonización (Donoso 1983, Otero 2006). Esto provocó el reemplazo de bosques nativos adultos por praderas y matorrales en valles y laderas de las cordilleras de la Costa y de Los Andes. En parte de estas áreas despejadas para la agricultura y ganadería y bosques destruidos por incendios, se han establecido mediante regeneración natural extensos renovales (Donoso, 1983), que abarcan 2,2 millones de hectáreas en el área de estudio (CONAF et al. 1999).

Otro cambio importante del bosque nativo ha sido su sustitución por plantaciones forestales de pinos y eucaliptos ocurrido a partir de 1974, después de la dictación del D.L. 701 que otorga bonificaciones a la forestación. Hacia 1970 se contaba sólo con 300.00 hectáreas de plantaciones (Lara y Veblen 1993), área que se expandió fuertemente hasta llegar a 2,4 millones de hectáreas en 2007 de las cuales 1,8 millones de hectáreas (75 \%) estaba cubierta por bosque nativo hacia 1550 (cuadro 3).
Bosques remanentes e implicancias para la conservación. Existen importantes diferencias regionales en los porcentajes remanentes de bosque nativo respecto al área existente hacia 1550 y de la proporción de dichos bosques que está protegido actualmente dentro del Sistema Nacional de Áreas Silvestres Protegidas del Estado (SNASPE). De los bosques remanentes en las regiones del Maule y del Biobío (22 \% y 36 \% del total original), sólo el 3,1 \% y $3,4 \%$, respectivamente, está protegido dentro del SNASPE (figura 2, Lara et al. 2006). La región de La Araucanía mantiene un $40 \%$ de sus bosques originales y el 23,9\% de ellos está incluido dentro del SNASPE, mientras que en las regiones de Los Ríos y Los Lagos en conjunto, dichos porcentajes son de $72 \%$ y 14,9 \%, respectivamente (figura 2, Lara et al. 2006). Lo anterior indica la necesidad de dar prioridad a la creación de nuevas áreas protegidas en las regiones de Maule y del Biobío a fin de mejorar el estado de conservación de los bosques nativos en dichas regiones.

Limitaciones de la información utilizada para la reconstrucción de la vegetación. Los documentos históricos analizados poseen semánticamente un amplio universo de análisis, por lo cual se encuentran limitados por su disponibilidad, subjetividad, ideales de cada época y selectividad. De esta manera, extensos territorios como el norte de La Araucanía, sur del Biobío, regiones lacustres cordilleranas de Los Ríos y Los Lagos, están descritos con menor detalle que la región del Maule en donde existió un temprano contacto entre españoles e indígenas (Camus 2006). A esto se suma la naturaleza de los datos, ya que si bien las descripciones se hacen más exhaustivas con el tiempo, no necesariamente se encuentran enfocadas hacia la cobertura boscosa o de la vegetación (Prieto et al. 2003). Por otro lado, los registros arqueológicos disponibles son fragmentarios, siendo escasas y poco actualizadas las síntesis culturales del período hacia 1550. Lo anterior dificulta una mejor comprensión de la transformación del paisaje por parte de las poblaciones picunche, mapuche, lafquenche costeras, huilliche y pehuenche, que habitaron desde la región del Maule hasta Chiloé.

A su vez, el análisis de la cobertura de la vegetación y uso de suelo hacia 1550 y de los cambios ocurridos entre ambas fechas, se ve limitado por la falta de continuidad de la información sobre los sucesivos procesos que van transformado el paisaje. Estos incluyen los reiterativos episodios de destrucción por causas humanas (incendios antropogénicos, tala, habilitación agropecuaria) o naturales (incendios naturales, erupciones volcánicas, deslizamientos de tierras, inundaciones) y la posterior recolonización de los bosques por procesos de sucesión ecológica. Tampoco se cuenta con los antecedentes suficientes para estimar los cambios en la distribución de los bosques, por ejemplo en su limite altitudinal, que puedan haber ocurrido debido a las fluctuaciones climáticas. 


\section{CONCLUSIONES}

A partir de un enfoque transdisciplinario que integra información proveniente de documentos históricos, sitios arqueológicos y la relación de estos registros, así como de la vegetación actual con variables ambientales, se desarrolló la primera reconstrucción de la cobertura boscosa y de la vegetación de la ecorregión de los bosques valdivianos lluviosos en Chile hacia 1550, expresada cartográficamente a escala 1: 500.000 .

Este estudio muestra la necesidad de continuar desarrollando una línea de investigación transdisciplinaria que, mediante la integración de métodos de investigación históricos, arqueológicos y biofísicos (vegetación potencial, palinología, dendrocronología), permita una mejor comprensión de los cambios ocurridos en la vegetación durante los últimos 450 años bajo fluctuaciones climáticas e intervención antrópica. El análisis de dichas transformaciones debe hacerse además en forma integrada al estudio de los cambios en los patrones de poblamiento humano, en las estrategias adaptativas de los pueblos originarios y de las sociedades europeas colonizadoras, las cuales han llevado a la transformación reiterada del paisaje de la ecorregión valdiviana en diversas direcciones.

\section{AGRADECIMIENTOS}

Esta investigación recibió el apoyo del proyecto FB 49 Universidad Austral de Chile-WWF (1998-1999) para la elaboración de una versión previa de este estudio, y de los proyectos FONDECYT No1090465, y del CRN II 2047-IAI-NFS Grant GEO-0452325. Se agradece a Patricio Rutherford, Oscar Thiers, Patricio Romero, Ramiro Trecaman y Aldo Farías por su apoyo en el procesamiento de la información, y a dos árbitros anónimos. La figura 1 fue elaborada por Aldo Farías.

\section{REFERENCIAS}

Abarzúa AM. 2009. Respuestas ambientales a cambios climáticos y culturales en la región de la Araucanía, Chile. Tesis Doctorado en Ciencias Forestales. Valdivia, Chile. Facultad de Ciencias Forestales y Recursos Naturales, Universidad Austral de Chile. 84 p.

Adán L, R Mera, F Bahamondes, S Donoso. 2007. Síntesis arqueológica de la cuenca del río Valdivia: proposiciones a partir del estudio de sitios alfareros prehispánicos e históricos. Revista Austral de Ciencias Sociales 12: 5-30.

Aldunate C. 1989. Estadio alfarero en el sur de Chile (500 a ca. 1800 dC). In Hidalgo J, V Schiappacasse, H Niemeyer, C Aldunate, I Solimano eds. Culturas de Chile, Prehistoria. Santiago, Chile. Andrés Bello. p. 329-348.

Armesto JJ, D Manuschevich, A Mora, C Smith-Ramirez, R Rozzi, AM Abarzúa, PA Marquet. 2010. From the Holocene to the Anthropocene: A historical framework for land cover change in southwestern South America in the past 15,000 years. Land Use Policy 27(2): 148-160.
Bengoa J. 2000. Historia del pueblo mapuche: siglo XIX y XX. Santiago, Chile. LOM. 430 p.

Bengoa J. 2003. Historia de los antiguos mapuches del Sur. Santiago, Chile. Catalonia. 528 p.

Camus P. 2006. Ambiente, bosques y gestión forestal en Chile. 1541- 2005. Santiago, Chile. LOM. 386 p.

Camus P, ME Solari. 2008. La invención de la selva austral. Bosques y tierras despejadas en la cuenca del río Valdivia (siglos XVI- XIX). Revista de Geografia Norte Grande 40: 5-22.

CONAF (Corporación Nacional Forestal, CL), CONAMA (Comisión Nacional del Medio Ambiente, CL), BIRF (Banco Internacional de Reconstrucción y Fomento), Universidad Austral de Chile, Pontificia Universidad Católica de Chile, Universidad Católica de Temuco. 1999. Catastro y evaluación de recursos vegetacionales nativos de Chile. Informe nacional con variables ambientales. $87 \mathrm{p}$.

CONAF (Corporación Nacional Forestal, CL), Universidad Austral de Chile. 2000. Monitoreo y Actualización de la Información de uso actual del suelo en la VII Región. 58 p.

CONAF (Corporación Nacional Forestal, CL), CONAMA (Comisión Nacional del Medio Ambiente, CL), Universidad Austral de Chile. 2008a. Catastro de uso del suelo y vegetación. Monitoreo y actualización región de los Ríos y provincias de Osorno y Llanquihue. Periodo 1998-2006. 19 p.

CONAF (Corporación Nacional Forestal, CL), CONAMA (Comisión Nacional del Medio Ambiente, CL), Universidad Austral de Chile. 2008b. Catastro de uso del suelo y vegetación. Monitoreo y actualización región de los Lagos. $19 \mathrm{p}$.

CONAF (Corporación Nacional Forestal, CL), CONAMA (Comisión Nacional del Medio Ambiente, CL), Universidad Austral de Chile. 2009. Catastro de uso del suelo y vegetación. Monitoreo y actualización región de la Araucanía. Periodo 1993-2007. 29 p.

Darwin Ch. 1945. Viaje de un naturalista alrededor del mundo. Buenos Aires, Argentina. El Ateneo. 618 p.

Dillehay T. 1990. Araucanía, presente y pasado. Santiago, Chile. Andrés Bello. 153 p.

Dillehay T. 2003. Interacción humana y ambiente: el desarrollo de kuel en Puren-Lumaco (región de La Araucanía). Revista Austral de Ciencias Sociales (Valdivia) 7: 17-25.

Dillehay T. 2007. Monuments, Resistance and Empires in the Andes: Araucanian Ritual Narratives and Polity. Cambridge, USA. Cambridge University Press. 484 p.

Dinerstein E, DM Olson, DJ Graham, AL Webster, SA Primm, MP Bookbinder, G Ledec. 1995. A conservation assessment of the terrestrial ecoregions of Latin America and the Caribbean. Washington, DC, USA. The World Bank. 129 p.

Donoso C. 1983. Modificaciones del Paisaje Chileno a lo largo de la historia. In Simposio Desarrollo y perspectivas de las disciplinas forestales en la Universidad Austral de Chile. Valdivia, Chile. Universidad Austral de Chile. p. 365-438.

Donoso C. 1993. Bosques Templados de Chile y Argentina. Santiago, Chile. Editorial Universitaria. 484 p.

Echeverría C, D Coomes, A Newton, JM Rey-Benayas, A Lara. 2007. Impacts of forest fragmentation on species composition and forest structure in the temperate landscape in southern Chile. Global Ecology and Biogeography 16: 426-439.

Elizalde R. 1968. La sobrevivencia de Chile. La conservación de sus recursos naturales. Ministerio de Agricultura, Servicio 
Agrícola y Ganadero. El Escudo Impresores Editores Ltda. Santiago, Chile. 492 p.

Guarda G. 2001. Nueva historia de Valdivia. Santiago, Chile. Ediciones Universidad Católica de Chile. 862 p.

González ME, TT Veblen, J Siebold. 2005. Fire history of Araucaria-Nothofagus forest in Villarrica Nacional park, Chile. Journal of Biogeography 32: 1187-1202.

Lara A, TT Veblen 1993. Forest plantations in Chile: a successful model? In Mather A ed. Afforestation. UK. Belhaven Press. p. 118-139.

Lara A, JC Aravena, S Fraver, A Wolodarsky. 1999. Fire and the dynamics of alerce (Fitzroya cupressoides) forests of Chile's Cordillera Pelada. Ecoscience 6: 100-109.

Lara A, V Sandoval. 2003 Generación de Cartografía y evaluación de recursos vegetacionales. In Oltremari J, KD Telen eds. Planificación de Áreas Silvestres Protegidas. Un manual para la planificación de áreas protegidas en Chile con especial referencia a áreas protegidas privadas. Santiago, Chile. CONAMA - FAO. p. 48-73.

Lara A, R Reyes, R Urrutia. 2006. Bosques. In Informe País, Estado del medio ambiente en Chile 2005. Santiago, Chile. Instituto de Asuntos Públicos. Centro de Análisis de Políticas Públicas. Universidad de Chile. p. 107-140.

Latcham R. 1928. La prehistoria chilena. Santiago, Chile. Universo. $238 \mathrm{p}$.

Mariño de Lobera P. 1865. Crónica del reino de Chile. Santiago, Chile. Imprenta El Ferrocarril. 456 p.

Miller A. 1976. The climate of Chile. In Schwerdtfeger W ed. World survey of climatology. Climates of Central and South America. Amsterdam, The Netherlands. Elsevier. p. 113-131.

Myers N, RA Mittermeier, CG Mittermeier, GA B. da Fonseca J Kent. 2000. Biodiversity hotspots for conservation priorities. Nature 403: 853-858.

Navarro X. 2004. Paisajes arqueológicos y territorialidad en la zona centro sur de Chile. Recuento actualizado de la historia prehispánica del área ubicada entre Tirúa y Valdivia. Cultura-Hombre-Sociedad 8: 71-86.

Otero L. 2006. La huella del fuego. Historia de los bosques nativos. Poblamiento y cambios en el paisaje del sur de Chile. Santiago, Chile. Pehuen. 171 p.

Philippi RA. 2003. El orden prodigioso del mundo natural. Santiago, Chile. Pehuen. 146 p.

Prieto MR, PE Villagra, NB Lana, EM Abraham. 2003. Utilización de documentos históricos en la reconstrucción de la vegetación de la Llanura de la Travesía (Argentina) a principios del siglo XIX. Revista Chilena de Historia Natural 76: 613-622.

Rosales D. 1989. Historia general del Reino de Chile. Flandes Indiano. Volumen 2. Santiago, Chile. Andrés Bello. 668 p.

Sanhueza L, F Vilches, C Rees, C Westfall, A Seelenfreund. 1994. Ocupaciones arqueológicas de la precordillera y cordillera de la cuenca del río Maule: un panorama general. In Actas del $2^{\circ}$ Taller de Arqueología de Chile Central. Consultado 8 feb. 2011. 17 p. Disponible en http://www.arqueologia.cl/ actas2/sanhuezaetal.pdf

Tellez E. 2004. Evolución histórica de la población mapuche del reino de Chile. 1536 -1810. Historia Indígena 8: 101126.

Treutler P. 1958. Andanzas de un alemán en Chile, 1851 - 1863. Santiago, Chile. Ed. del Pacífico. 570 p.

Torrejón F, M Cisternas. 2002. Alteraciones del paisaje ecológico araucano por la asimilación mapuche de la agroganadería hispano-mediterránea (siglos XVI-XVII). Revista Chilena de Historia Natural 75: 729-736.

Veblen TT, DH Ashton, FM Schlegel. 1979. Tree regeneration strategies in a lowland Nothofagus- dominated forest in south-central Chile. Journal of Biogeography 6: 329-340.

Vivar J. 1979. Crónica y relación copiosa y verdadera de los reynos de Chile. Berlín, Alemania, Colloquium Verlag. 232 p. 
